\title{
Efficient Tumor Detection in MRI Brain Images
}

\author{
https://doi.org/10.3991/ijoe.v16i13.18613 \\ Y. Sri Lalitha ${ }^{(凶)}$, Katapally Manognya, Pabba Keerthana \\ Mudunuri Vineetha \\ Gokaraju Rangaraju Institute of Engineering and Technology, \\ Hyderabad, India \\ srilalitham.y@gmail.com
}

\begin{abstract}
Detection of brain of tumor is a laborious task as it involves identification, segmentation followed by detection of the tumor. It is a very challenging task to envisage uncommon structures in the image of human brain [15]. An Image processing concept called MRI can be used to visualize different structures of human body. The Magnetic Resonance images (MRI) are used to detect the uncommon portions of human brain. This paper explores different noise removal methods accompanied by Balance-contrast enhancement technique (BCET) which results in increased accuracy. Segmentation followed by canny edge detection is performed on the improved images to detect the fine edges of the abnormalities present. The model attained an accuracy of at most $98 \%$ in detecting the tumor or the abnormality in a human brain which determines the effectiveness of the proposed model.
\end{abstract}

Keywords-Brain Tumor, MRI Image, Fuzzy Clustering

\section{Introduction}

The National Cancer Institute (NCI) predicted that 22,070 new instances of brain and different vital apprehensive device (CNS) cancers might be diagnosed inside the US in 2009. Masses of peculiar or abnormal portion in our human brain is defined as tumor. The skull which surrounds human brain could be inflexible. These sorts of restrained parts can result in difficulties. They may be malignant and benign. While these cancerous/ noncancerous tumors increase in the size, they result in the increase of size of the skull. This might cause reason harm to a person by increasing the mortality rate. Today, maximum clinical establishments use the World Health Organization (WHO) type device to distinguish brain tumors. The WHO classifies brain tumors by means of cellular beginning and the way those cells behave. Our approach is to create a model to determine the brain tumors in an appropriate and efficient way which could be used by the medical practitioners to ease their task. 


\section{Background Study}

A set of odd cells in the brain which might additionally cause cancers are brain tumors. This is a prime leading motive of demise and liable for around $11 \%$ of all deaths which take place globally. The cancer's incidence detection price is developing in the global scale. Hence, its detection is important in advance range. The existing models for the detection of brain tumors are classified into contour and region specified methods. Region specified methods focus on the pixel clusters and select those of them which has greater incidence of similarities. These methods operate on automating the low-level functions like the Classification, Thresholding, Analysis of histogram etc. Numerous researchers like Dou [13], Spotlighted the regional information of individual pixel and ignored the data on boundary and shape. In 2017, Verma [11] has proposed a model for segmenting medical images using the morphological operators accompanied by the threshold collection. In 2019, Samir Abou [14] used a mixed reality system to merge the digital image of the patient anatomy with the patient visual image. While Rajesh [12] in 2014 has done the same followed by the watershed method and LoG edge maps. The purpose of this paper is to solve the complication of distinguishing the abnormal brain cells from the normal and healthy cells using optimised segmentations of the styles are intuitive. However, we invite you to read carefully the brief description below.

\section{Implementation}

\subsection{System architecture}

The proposed model follows the phases of a Pattern recognition steps depicted flow graph in the Figure below: 


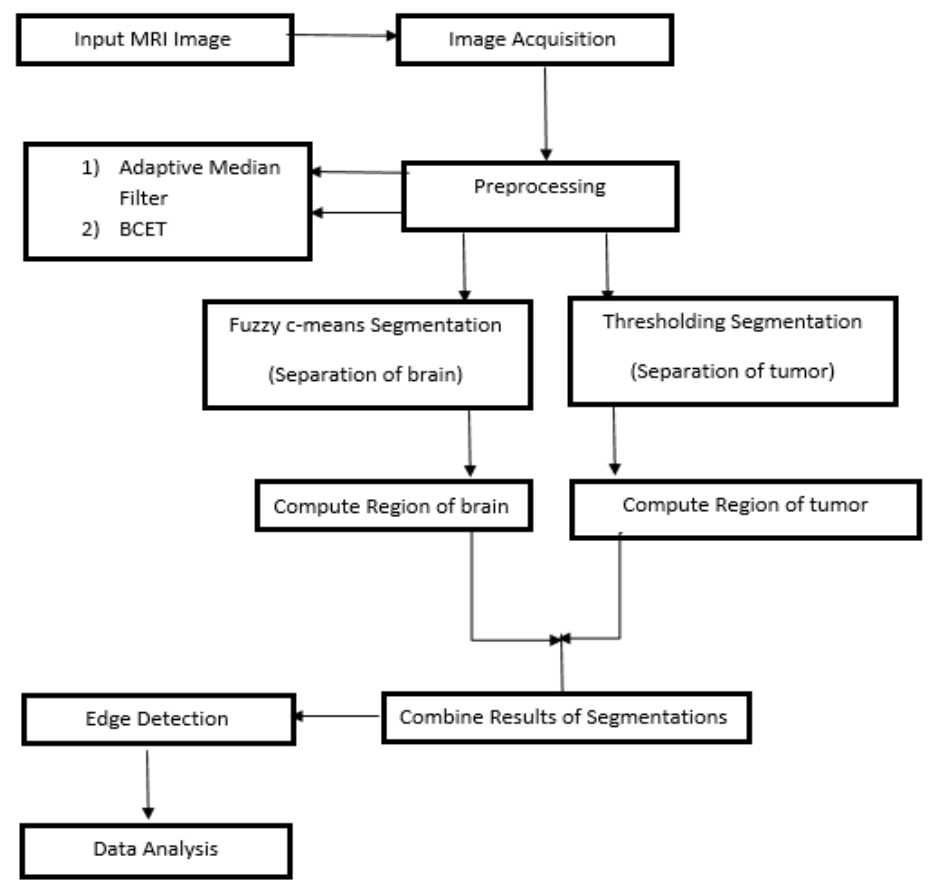

Fig. 1.

\subsection{Image acquisition}

Digital Imaging and Communication on Medicine (DICOM) and Brain Web datasets were considered. In which, 15 images of tumor-infected tissues were taken from DICOM dataset. The Brain Web dataset consisted of simulated 3D brain magnetic resonance data, in this 14 out of 47 of tainted brain tissues were included.

The dataset consisting of patients MRI images are of Color, Grayscale or intensity images. Each image in the dataset is of size 256X256.

It is supposed that the MRI images are Greyscale images and if they are colored, the images are converted into Greyscale image followed by a size conversion into $256 \times 256$. The values between 0 to 255 are used to represent the converted images in which 0,255 corresponds to black and white respectively.

\subsection{Preprocessing}

Improvement of the images for accurate diagnosis is the elementary task of preprocessing module. Improvement of the aspects like the signal to noise ratio and the enhancement of images are also taken care of. This includes the elimination of the irrelevant elements in background accompanied by the smoothening of image. The work here uses Adaptive Median Filter and Balance Contrast Enhancement Filter [3][6]. 
1. Adaptive median filter: This filter removes noise by classifying pixels. That is, it analyses each pixel by comparing it to its surrounding pixels and if it is contrasting from its majority of the neighboring pixels, it is considered as noise. Median pixel value from the neighborhood is utilized to replace the noise [5].

2. Balance contrast enhancement technique: The area of interest is highlighted for exceptional understanding and better detection of tumor region which can be carried out using Balance contrast enhancement technique (BCET). The tumor part is highlighted in compressed white pixel.

3. Segmentation: Segmentation is essential in order to decide the appropriate treatment to be given. Its result consists of the set of regions which collectively form the entire brain as is visualized as contrast images. This module works with two algorithms namely Fuzzy C means and Thresholding [4].

\section{a) Fuzzy Clustering}

This algorithm is used to distinguish the segment of normal brain by performing clustering which involves the iterative search for fuzzy cluster sets.

The algorithm assigns all the related data points based on the degree of membership using a distance measure from the centroids of each cluster, so if the memberships of a particular data point are added together it will sum up to one.

The entire objective of Fuzzy C Means is to minimize the following objective function.

$$
j_{m}=\sum_{k=1}^{A} \sum_{L=\perp}^{B} z_{i j}^{n}\left\|p_{i}-q_{j}\right\|^{2} ; 1 \leq n<\infty
$$

where $z_{i j}$ is the degree of membership of $p_{i}$ in cluster $L$, and $q_{j}$ is the cluster center where $\mathrm{n}>1$, updating $\mathrm{z}_{\mathrm{ij}}$ and $\mathrm{q}_{\mathrm{j}}$ is done by following formulation

$$
\begin{gathered}
z_{i j}=\frac{1}{\sum_{r=1}^{B}\left(\frac{\left\|p_{i}-q_{r}\right\|}{\left\|p_{i}-q_{r}\right\|}\right)^{\frac{2}{m-1}}} \\
q_{j}=\frac{\sum_{i=1}^{A} z_{i j}^{m} \cdot p_{i}}{\sum_{i=1}^{A} z_{i j}^{m}}
\end{gathered}
$$

After each iteration of assigning membership to all the data points, $q_{l}$ and $z_{k l}$ are updated.

$$
\max _{k l}\left\{\left|z_{k_{l}}^{(x+1)}-z_{k l}\right|\right\}<\varepsilon
$$

The process of updating and iterating halts when the condition in Eq-4 is met, the termination criterion is reached, when $0 \leq \mathcal{E} \leq 1$, whereas $\mathrm{k}$ represents the number of iterations. 


\section{Algorithm Steps:}

Step 1: Randomly cluster centroids are selected

Step 2: Calculate the fuzzy membership $\mathrm{z}_{\mathrm{ij}}$ using $\mathrm{Eq}-2$ for each data point in the dataset with respect to each cluster centroid.

Step 3: Update the cluster centroids using Eq -3

Step 4: Repeat Step 2 and Step 3 until $\mathrm{Eq}-4$ condition is not met or minimum $\mathrm{j}_{\mathrm{m}}$ is achieved.

b) Thresholding

The greyscale image is converted into a binary image. The crucial part is to set a threshold value and the pixels whole value exceed the threshold value are selected and turned into white whereas the other pixels are turned into black. This enhanced region has been cropped and edge detection is performed. Using canny edge detection algorithm.

4. Contour representation: Different algorithms such as Prewitt, LoG and Canny were used to detect the edges resulting in the generation of contour map. In the proposed methodology Canny edge detector proved to be most effective of all. With the help of the canny edge detection algorithm, it detects the edges of the pixels and highlights the tumor region.

\section{$4 \quad$ Experimental Results}

The Observation results clearly shows the identification of tumor is quick and precise when compared to the other clinal methods. The proposed model achieved swift and accurate detection of tumors. Table 1 contains the performance analysis carried out on different edge maps.

To measure the efficiency and accuracy of the edge-detection map in the proposed model, a comparison study has been done and the factors considered are sensitivity, Accuracy and Err [7-10].

$$
\text { Sensitivity_Score: } \frac{T P}{T P+F N}
$$

[TP: True Positive, FN: False Negative]

$$
\text { Accuracy_Score: } \frac{T P+T N}{T P+T N+F N+F P}
$$

[TN: True Negative, FP: False Positive]

Err $=$ Percentage of erroneous pixels

Table 1. Edge Maps Comparison

\begin{tabular}{|l|c|c|c|}
\hline & Err & Sensitivity & Accuracy \\
\hline Proposed & 0.4325 & 0.8783 & 0.9812 \\
\hline Prewitt & 0.6720 & 0.3347 & 0.9559 \\
\hline LoG & 0.7625 & 0.223 & 0.9218 \\
\hline
\end{tabular}


In the Figure below the comparison between edge detecting maps has been shown and the proposed model allowed us to obtain a high accuracy on an average of $4-7 \%$ over the other maps by detecting less erroneous edges.

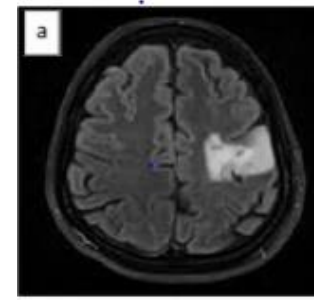

a) Input MRI Image

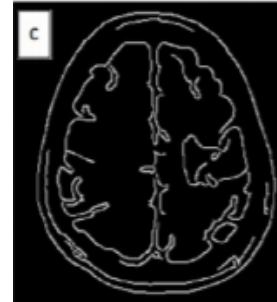

b) Canny

(Proposed)

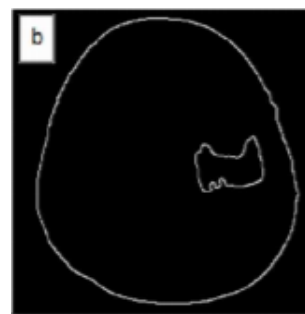

c) Prewitt

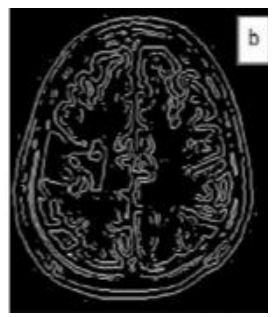

d) Laplacian of Gaussian Edge Method

Fig. 2.

The Adaptive median Filter, BCET have been used for noise removal and image enhancement as it has been found to work better with our proposed model compared to mean filter, GrayScale, Adaptive median, Weiner filter, Gaussian filter.

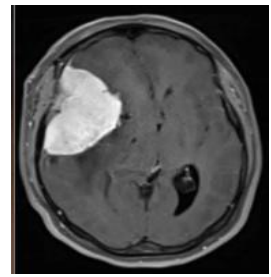

Grayscale Image

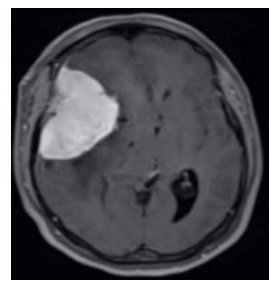

Weiner Filter

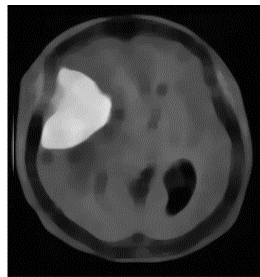

Adaptive median filter

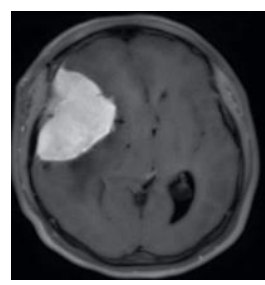

Gaussian Filter

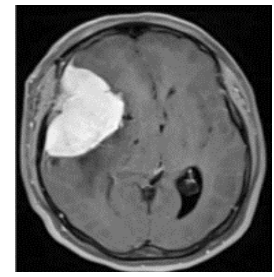

BCET

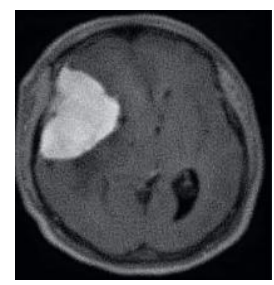

Mean Filter

Fig. 3. 
Noise Removal Filters

Fuzzy $\mathrm{C}$ means and Thresholding has been applied on image after filtering. Image filtered using AMF has been used as an input for Fuzzy $\mathrm{C}$ means and the image filtered using BMF has been used as an input for Thresholding. Then results of Fuzzy $\mathrm{C}$ means and Thresholding stages has been taken as input for segmentation stage.

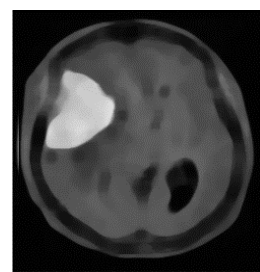

a) Input for Fuzzy $\mathrm{C}$ means

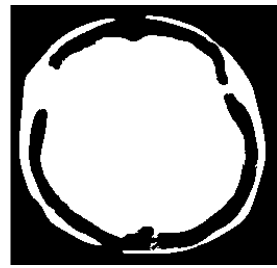

b) Output of Fuzzy C means

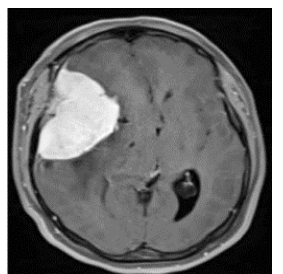

c) Input for Thresholding

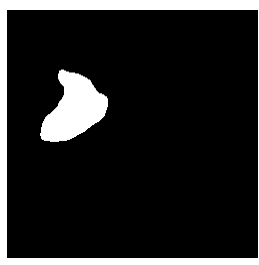

a) Output of Thresholding

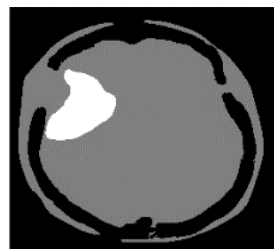

b) Segmentation result

Fig. 4.

The edge detection and contour representation are done to clearly show the infected region for the understanding of all masses of audience.

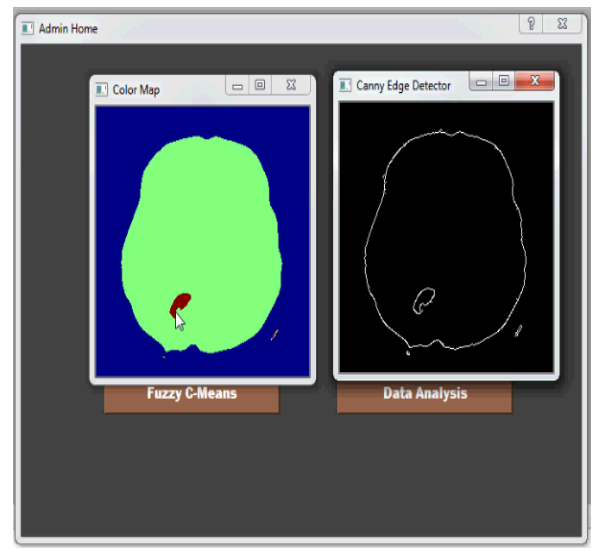

Fig. 5. Contour representation \& Edge Detection Results 


\section{Comparative study of performance of edge detection algorithms}

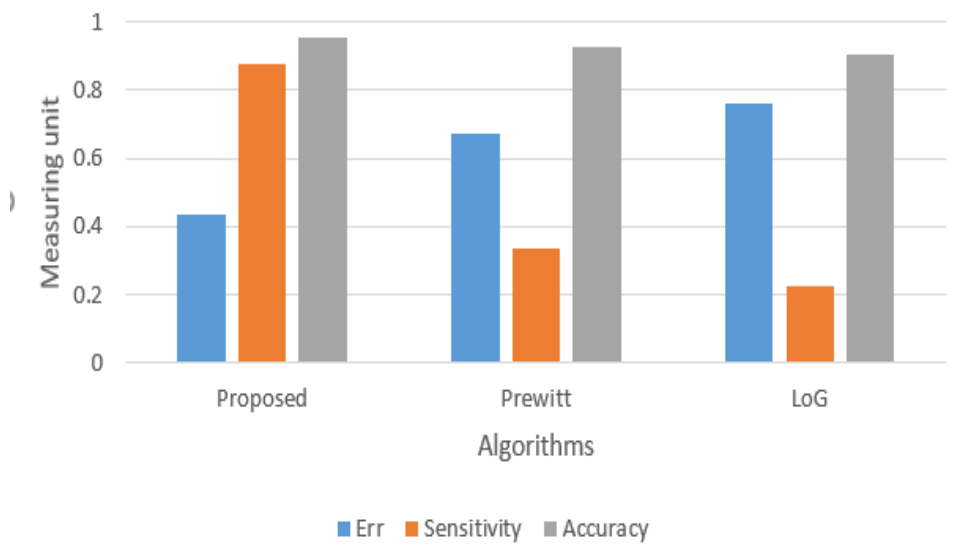

Fig. 6.

\section{Conclusion}

In this paper, An MRI picture of a human brain is considered for tumor detection. Segmentation has been improved to detect edges of the tumor to achieve utmost accuracy in identifying effected region of the brain (human). The Contour map is used to differentiate the tumor. Comparative study has been done to choose the best filter and the edge detection algorithm which provided with finest results.

\section{Future Work}

This model can be integrated with some object detection applications. In a depth image, for smaller objects to be detected, the images must contain minimal noise and accurate edges which can obtained by using the proposed model. This model can also be made available to all the medical practitioners and can be integrated with the available health apps where a patient can also access the option of self-problem detection and get notified if problem persists.

\section{$7 \quad$ References}

[1] Manju (2015), A Survey on Brain Tumor Detection Technique, in International Journal of Computer Science and Management Studies. 
[2] Kapoor, A survey on brain tumor detection using image processing techniques. In 7th International Conference on Cloud Computing, Data Science \& Engineering Confluence. https:// doi.org/10.1109/confluence.2017.7943218

[3] Anoop B. K. (2017), Comparison of different image pre-processing methods used for retinal fundus images. https://doi.org/10.1109/icedss.2017.8073677

[4] Nayak, P., Devulapalli, A. (2016), A Fuzzy Logic-Based Clustering Algorithm for WSN to Extend the Network Lifetime, IEEE Sensors Journal, 16 (1), article number 7222367, pp. 137-144. https://doi.org/10.1109/jsen.2015.2472970

[5] Vasicek Z and L. Sekanina (2008), Novel Hardware Implementation of Adaptive Median Filters, 11th IEEE Workshop on Design and Diagnostics of Electronic Circuits and Systems, Bratislava. https://doi.org/10.1109/ddecs.2008.4538766

[6] Swaraj K. (2017) Protection of medical image watermarking, Journal of Advanced Research in Dynamical and Control Systems, 9 (Special issue 11), pp. 480-486.

[7] Hassan (2015), Detecting Brain Tumour from MRI Image using Matlab GUI Programme in International Journal of Computer Science \& Engineering Survey Vol.6. https://doi.org/ $\underline{10.5121 / \text { ijcses.2015.6604 }}$

[8] Jain (2013), Review of Image Classification Methods and Techniques, International Journal of Engineering Research \& Technology.

[9] Varun Shree (2018), Identification and classification of brain tumor MRI images with feature extraction using DWT and probabilistic neural network, Brain Informatics, Springer. https://doi.org/10.1007/s40708-017-0075-5

[10] Y. Sri Lalitha, Dr. A. Govardhan (2013), Semantic Framework for Text Clustering with Neighbor, ICT and Critical Infrastructure: Proceedings of the 48th Annual Convention of CSI, Volume II, Advances in Intelligent Systems and Computing 249, (C) Springer International Publishing Switzerland pp.261-271. https://doi.org/10.1007/978-3-319-03095-1_29

[11] Priya, Vivek Singh Verma (2017) New morphological technique for medical image segmentation, 3rd International Conference on Computational Intelligence \& Communication Technology (CICT), Ghaziabad. https://doi.org/10.1109/ciact.2017.7977282

[12] Rajesh (2012), Brain Tumour Extraction from MRI Images Using-MATLAB in International Journal of Electronics, Communication \& Soft Computing Science and Engineering, 2012.

[13] Weibei Dou (2006), A framework of fuzzy information fusion for the segmentation of brain tumor tissues on MR images, Elsevier.

[14] Samir Abou El-Seoud, Amr S. Mady, Essam A. Rashed (2019), An Interactive Mixed Reality Ray Tracing Rendering Mobile Application of Medical Data in Minimally Invasive Surgeries in International Journal of Online and Biomedical Engineering (iJOE). https://doi.org/ 10.3991/ijoe.v15i06.9933

[15] Mohammad Rashid Hussain (2019) A New Neuro Informatics Approach to Optimize Diagnosis Cost in Neurology: An Operational Research Tool, in International Journal of Online and Biomedical Engineering (iJOE). https://doi.org/10.3991/ijoe.v15i06.10141 


\section{Authors}

Dr. Y. Sri Lalitha is a Professor, Department of IT, Gokaraju Rangaraju Institute of Engineering and Technology, JNTU(H), Hyderabad, India. Ph.D. from ANU, Guntur, M. Tech. (CS) from JNTU(H) has around 15 publications in renowned Journals. Her Areas of Research Interests includes Machine Learning, NLP, Data Science and Big Data Analytics.

Katapally Manognya is a Studying Final B. Tech Information Technology, Gokaraju Rangaraju Institute of Engineering and Technology, JNTU(H), Hyderabad, India.

Pabba Keerthana is a Studying Final B. Tech Information Technology, Gokaraju Rangaraju Institute of Engineering and Technology, JNTU(H), Hyderabad, India.

Mudunuri Vineetha is a Studying Final B. Tech Information Technology, Gokaraju Rangaraju Institute of Engineering and Technology, JNTU(H), Hyderabad, India.

Article submitted 2020-09-16. Resubmitted 2020-10-23. Final acceptance 2020-10-28. Final version published as submitted by the authors. 